\title{
Granulomatous angiitis of the central nervous system: a case with recurrent intracerebral haemorrhage
}

\author{
RE CLIFFORD-JONES, S LOVE, N GURUSINGHE \\ From The National Hospital for Nervous Diseases, Queen Square, London, UK
}

SUMMARY A 43-year-old man with a history of radiculomyelopathy developed recurrent intracerebral haemorrhage confirmed by CT brain scan. Cerebral angiography and systemic investigations showed no underlying cause for the haemorrhage. Cerebral biopsy at the time of evacuation of a haematoma revealed granulomatous angiitis. This condition may be responsive to steroids and should be considered in cases of intracerebral haemorrhage of obscure origin.

Granulomatous angiitis of the central nervous system (CNS) is an uncommon form of vasculitis, first delineated as a distinct disease entity by Cravioto and Feigin in 1959.' The disorder is largely confined to the CNS and may involve cerebral hemispheres, cerebellum, brain stem, or spinal cord and roots. ${ }^{1-6}$ Most cases have a fatal outcome $e^{411}$ and a tissue diagnosis has rarely been established in life. ${ }^{2379} \mathrm{We}$ describe an unusual case, diagnosed by brain biopsy, with a relapsing and remitting course which included recurrent intracerebral haemorrhage.

\section{Case history}

In February 1982 a 43-year-old man presented with a two month history of pain and numbness in the left leg and three brief episodes of double vision. Abnormal signs were confined to the left leg; the calf muscles were weak with a depressed ankle jerk. The left plantar response was extensor and there was hypalgesia over the left S1 dermatome. Routine biochemical and haematological studies were normal. A chest radiograph and lumbar radiculogram showed no abnormalities, but the CSF protein was raised $(1.7 \mathrm{~g} / \mathrm{l})$. His condition remained unchanged until April when over ten days he became unable to walk and developed sphincter disturbance. On examination he had a palpable bladder and a severe flaccid paraparesis with an absent left knee jerk, depressed left ankle jerk and absent plantar responses. Cutaneous sensation was impaired in the left L2-S5 and the right S3-S5 dermatomes.

Address for reprint requests: Dr RE Clifford-Jones, The National Hospital for Nervous Diseases, Queen Square, London WC1N 3BG, UK.

Received 30 November 1984 . Accepted 9 February 1985
Haematological and radiological investigations including myelography were again normal but the CSF now con-otained 163 lymphocytes $/ \mathrm{mm}^{3}$, and a transudate of $2 \mathrm{~g} / \mathrm{l}$ of protein. No fungi or bacteria were cultured. Serum titres of antibodies to viruses including herpes simplex and zoster,, the visual evoked potentials, and a CT head scan were normal.

The patient was started on $16 \mathrm{mg}$ of dexamethasone daily and his condition improved so that by discharge fous weeks later he had normal sphincter control and onlye minimal weakness of the left foot. Steroids were stopped after three weeks. A subsequent Kveim test was negative and the serum angiotensin converting enzyme level was normal. In July steroids were restarted because the patient developed diplopia due to impaired abduction of the right eye. The diplopia resolved and the dose of steroids was reduced. In January 1983, whilst on $2.5 \mathrm{mg}$ of prednisolone, the patient experienced intermittent diplopia. He soon developed right-sided headaches with repeated vomiting. Examination showed a drowsy, dysarthric patient with a stiff neck. There was a left facial weakness and a profound left hemiparesis with sensory loss. A CT scan demonstrated a right inferior frontal haematoma with blood in the ventricles. Angiography revealed no source for the haemorrhage. The patient was given steroids and managed supportively. He improved markedly over the next two weeks and was discharged with only a mild left hemiparesis and off all treatment. In May 1983 he presented with a sudden, severe headache and pain behind the right eye. He was dysarthric, with a left homonymous hemianopia, a left sided sensory loss involving the face and a mild left hemiparesis. A CT scan showed a haematoma in the right parietal lobe. The next day after a generalised convulsion the patient became densely hemiplegic and lapsed into coma. A repeat CT scan showed extension of the haemorrhage with considerable mass effect, but 
arteriography again failed to show any source for the haemorrhage. An emergency craniotomy was carried out and the haematoma evacuated. Several small biopsy specimens were taken of the surrounding leptomeninges and brain, which was grey and granular.

\section{Histology}

The biopsied fragments were processed to paraffin wax, sectioned at 5 or $10 \mu \mathrm{m}$ and stained by routine histological methods which included stains for parasites, fungi and acid-fast bacilli. Within the leptomeninges were several small veins and arteries, surrounded by lymphocytes, epithelioid macrophages and occasional giant cells (fig a). Many small cortical blood vessels had undergone fibrinoid necrosis (fig b). A few medium-sized arteries in the cortex and superficial white matter were surrounded by mononuclear inflammatory cells and extravasated fibrinoid material. Surrounding brain parenchyma was oedematous and disrupted by haemorrhage. Stains for organisms were negative. A diagnosis was made of granulomatous angiitis.

\section{Post-operative course}

The patient made a good recovery and was discharged home on steroids and anticonvulsants. In November 1983 the medication was changed to cyclophosphamide $(2 \mathrm{mg} /$ $\mathrm{kg}$ ) and alternate day steroids, but the cyclophosphamide

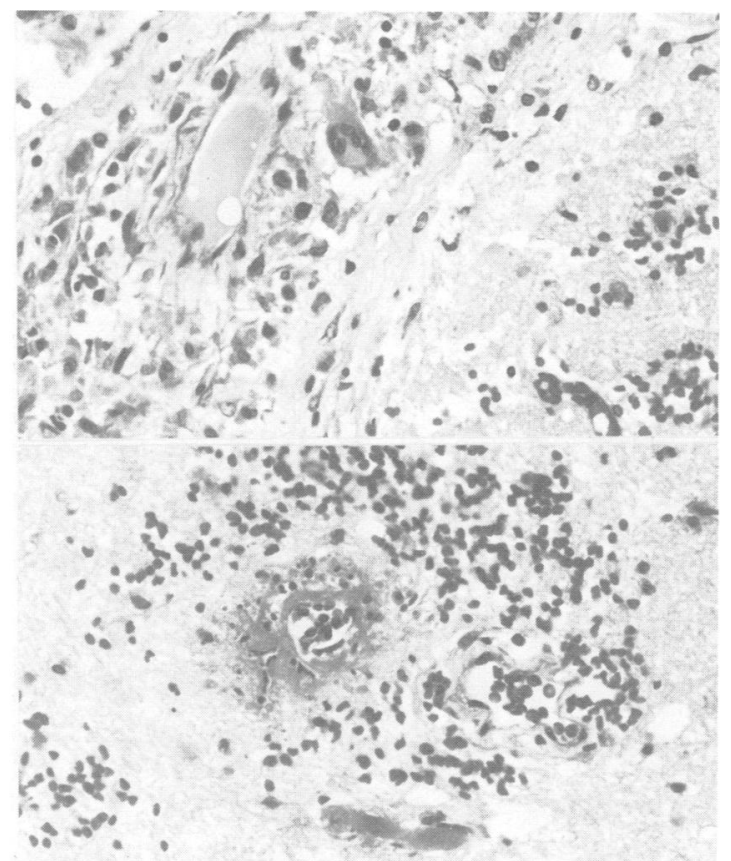

Fig (a): Section through a sulcus and adjacent cerebral cortex. Leptomeningeal blood vessels are surrounded by lymphocytes and macrophages. The infiltrate includes a multinucleated giant cell. (b): Fibrinoid necrosis of blood vessels in the superficial white matter. Surrounding white matter is oedematous and disrupted by haemorrhage. (Haematoxylin and eosin $\times 450$.) had to be discontinued because of bone marrow depression. The patient remains on alternate day steroids and has had no further neurological episodes except for infrequent fits over the 14 months since his operation.

Investigations have failed to show any evidence of systemic disease, and levels of auto-antibodies, C-reactive protein, complement, immune complexes and immunoglobulins have been persistently normal.

\section{Discussion}

Granulomatous angiitis is usually readily distinguished from infective granulomatous diseases of the central nervous system but histological differentiation from neurosarcoidosis may be difficult. ${ }^{10}$ The cerebral biopsy of this patient demonstrated a noncaseating, granulomatous infiltrate in the leptomeninges and surrounding many small blood vessels. Other blood vessels showed fibrinoid necrosis and, often, thrombosis. Urich ${ }^{10}$ suggested that neurosarcoidosis could be excluded in the absence of evidence of systemic disease and in the presence of necrotising vasculitis separate from granulomatous lesions, as in the present case. In some reports granulomatous angiitis has been associated with herpes zoster infection ${ }^{589}$ or with lymphoproliferative disease ${ }^{378}$ but in this patient there was no evidence of either condition.

The initial radiculomyelopathy and the subsequent intracerebral haemorrhages all caused extensive neurological deficits but on each occasion the patient recovered almost completely. It seems probable that the use of steroids on each occasion contributed to the recovery. Previous cases of granulomatous angiitis treated with steroids have shown temporary improvement in some instances and as a group have tended to survive longer than untreated cases. ${ }^{41}$ Remission of histologically proven granulomatous angiitis has been reported in two patients; ${ }^{2} 3$ in one steroids were given with additional treatment for an associated lymphoma and in the other remission only occurred when cyclophosphamide was added.

Computed tomography of our patient demonstrated recurrent intracerebral haematomas rather than the low density ischaemic lesions ${ }^{3}$ or normal scans $^{2}$ described in other cases. Angiography on two occasions showed no source for the haemorrhage and the diagnosis was made following an emergency craniotomy. Significant intracerebral haemorrhage in granulomatous angiitis has previously been recognised only as a preterminal event. ${ }^{5}$

This case illustrates that granulomatous angiitis should be considered as a cause of intracerebral haemorrhage, particularly when associated with relapsing and remitting disease of the CNS. Long- 
term administration of steroids may reduce the likelihood of further haemorrhage.

We thank Sir Roger Bannister and Mr R Hayward for permission to report details of the patient, and Mr and Mrs JM Mills for help with the photography.

\section{References}

' Cravioto H, Feigin I. Noninfectious granulomatous angiitis with a predilection for the nervous system. Neurology (Minneap) 1959;9:559-609.

${ }^{2}$ Cupps TR, Moore PM, Fauci AS. Isolated angiitis of the central nervous system. Prospective diagnostic and therapeutic experience. Am J Med 1983;74:97-105.

${ }^{3}$ Greco FA, Kolins J, Rajjoub RK, Brereton HD, Hodgkin's disease and granulomatous angiitis of the central nervous system. Cancer 1976;38:2027-32.

${ }^{4}$ Harrison PE. Granulomatous angiitis of the central nervous system. J Neurol Sci 1976;29:335-41.
${ }^{5}$ Kolodny EM, Rebeiz JJ, Caviness VS, Richardson EP. Granulomatous angiitis of the central nervous system. Arch Neurol (Chic) 1968;19:510-24.

- Nurick S, Blackwood W, Mair WGP. Giant cell granulomatous angiitis of the central nervous system. Brain 1972; 95: 133-42.

${ }^{7}$ Rewcastle NB, Tom MT. Non-infectious granulomatous angiitis of the nervous system associated with Hodgkin's disease. J Neurol Neurosurg Psychiatry 1962; 25:51-58.

${ }^{8}$ Rosenblum WI, Hadfield MG. Granulomatous angiitis of the nervous system in cases of herpes zoster and lymphosarcoma. Neurology

(Minneap) 1972;22:349-53.

${ }^{9}$ Rosenblum WI, Hadfield MG, Young HF. Granulomatous angiitis with varicella zoster. Ann Neurol 1978; 3:374-5.

${ }^{10}$ Urich H. Neurosarcoidosis or granulomatous angiitis: a problem of definition. Mt Sin J Med 1977;44:718-25.

$"$ Vincent FM. Granulomatous angiitis. $N$ Eng $J$ Med 1977;296:452. 